\title{
Recursive Partitioning Assessment
}

National Cancer Institute

\section{Source}

National Cancer Institute. Recursive Partitioning Assessment. NCI Thesaurus. Code C102857.

A set of pretreatment prognostic factors used to predict potential outcomes for neurooncology patients. 